\title{
Langmuir probe characterization of laser ablation plasmas
}

\author{
Brendan Doggett ${ }^{\mathrm{a})}$ and James G. Lunney \\ School of Physics, Trinity College, Dublin 2, Ireland
}

(Received 8 May 2008; accepted 18 November 2008; published online 6 February 2009)

\begin{abstract}
For laser ablation plumes that are significantly ionized, Langmuir probes have proved to be a useful tool for measuring the plume shape, ion energy distribution, and electron temperature. Typically in laser ablation plasmas the flow velocity is supersonic, which complicates the interpretation of the current-voltage probe characteristic. In this paper we describe some recent developments on the application of Langmuir probes for laser ablation plume diagnosis. We have investigated the behavior of the probe when it is orientated perpendicular, and parallel, to the plasma flow, and show how an analytical model developed for plasma immersion ion implantation, can quantitatively describe the variation of the ion current with probe bias for the case when the plasma flow is along the probe surface. The ion signal recorded by a probe in the parallel position is proportional to the ion density and the square root of the bias voltage. It is shown that the current varies as $m_{i}^{-1 / 2}$ so that by comparing the ion signals from the parallel and perpendicular positions it is possible to estimate the mass of the ions detected. We have also determined the temporal variation of electron temperature. A planar probe oriented parallel to the plasma flow, where the ion current due to the plasma flow is eliminated, gives a more reliable measurement of $T_{e}(<0.6 \mathrm{eV})$. The measured $T_{e}$ is consistent with the measured ion current, which is dependent on $T_{e}$ when the time taken for an element of plasma to traverse the probe is longer than the time taken for the matrix ion sheath extraction phase. (C) 2009 American Institute of Physics. [DOI: 10.1063/1.3056131]
\end{abstract}

\section{INTRODUCTION}

Langmuir probes have been widely used to diagnose the low temperature plasmas produced by laser ablation for pulsed laser deposition of thin films. ${ }^{1,2}$ These probes have been used to measure the plasma density and temperature, the plasma flow velocity and the shape of the ablation plume expansion. ${ }^{3}$ Typically for laser ablation in vacuum, the plasma expansion is supersonic, thus the plasma flow velocity is much greater than the Bohm velocity, given by

$$
v_{B}=\sqrt{\frac{k T_{e}}{m_{i}}},
$$

where $T_{e}$ is the electron temperature and $m_{i}$ is the ion mass. For a planar probe oriented normal to the plasma flow, and biased negatively to reject electrons, the ion current will be dominated by the ion flux due to the flow. For large negative bias, $V \gg k T_{e} / e$, the magnitude of the ion signal from a planar probe does not depend on the bias. The ion velocity can be determined from the ion time of flight (TOF) from the target to the probe and hence the ion density can be found from the magnitude of the ion signal. With regard to measuring the current due to electrons, it should be noted that the ion velocities correspond to energies of up to $200 \mathrm{eV}$, and so the ion flow is not much influenced by small values of positive bias of a few volts.

This paper reports the results of an investigation of some aspects of the behavior of a planar Langmuir probe in a laser produced plasma (LPP). We compare the behavior of the probe when it is orientated perpendicular and parallel to the

\footnotetext{
${ }^{a)}$ Electronic mail: doggettb@gmail.com.
}

plasma flow. We present an adaptation to an analytical model developed for plasma immersion ion implantation (PIII), to quantitatively describe the variation of the ion current with probe bias for the case when the plasma flow is along the probe surface.

\section{PROBE THEORY}

The application of probe theories has been developed for specific probe geometries and plasma regimes. ${ }^{4}$ The regimes are defined by the relative values of the probe dimensions, the sheath thickness, and the values of the mean free path for the various collisions among electrons and ions. Away from the probe surface, any departure from quasineutrality can only exist over distances less than the Debye length, $\lambda_{D}$, given by

$$
\lambda_{D}=\sqrt{\frac{\varepsilon_{0} k T_{e}}{e^{2} n_{e}}} .
$$

Typically at several centimeters from the target in a LPP, $n_{e} \approx 10^{17} \mathrm{~m}^{-3}$ and $k T_{e} \sim 0.3 \mathrm{eV}$, making $\lambda_{D} \sim 13 \mu \mathrm{m}$. The planar Langmuir probes used in LPPs have transverse dimensions of $1-10 \mathrm{~mm}$. An initial estimate of the sheath thickness, $h_{0}$, can be found using the formula for the highvoltage sheath with a uniform ion density, $n_{i}$, known as a matrix sheath: $h_{0}=\sqrt{2 \varepsilon_{0} V / e n_{i}}$. For a bias voltage of $10 \mathrm{~V}$ and a typical ion density of $10^{17} \mathrm{~m}^{-3}, h_{0}=0.1 \mathrm{~mm}$. The mean free paths for ion-ion $\left(\lambda_{i i}\right)$, electron-electron $\left(\lambda_{e e}\right)$ and electron-ion collisions $\left(\lambda_{e i}\right)$ can be estimated; ${ }^{5}$ for a singly ionized Ag plasma with $n_{i}=n_{e}$ and $k T_{i}=k T_{e}=0.3 \mathrm{eV}$, the values are $\lambda_{i i}=14 \mathrm{~mm}$ and $\lambda_{e e}=\lambda_{e i}=12 \mathrm{~mm}$. Thus we can see that $\lambda_{i i, e i}>d \gg h_{0}$. Under these conditions, collisionless 

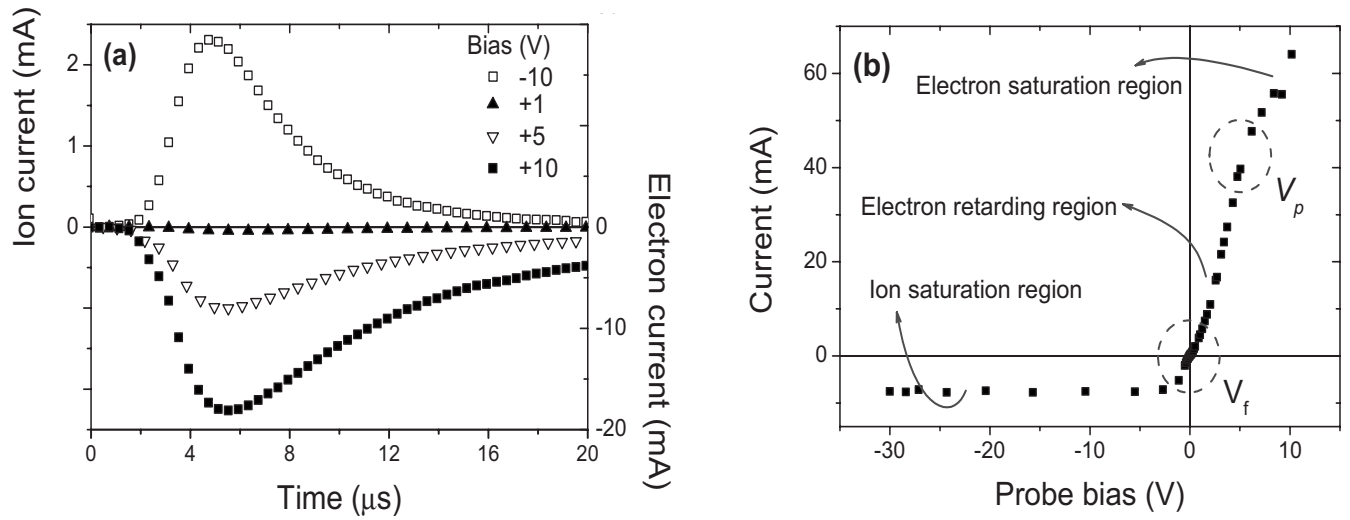

FIG. 1. (a) Typical example of the ion and electron current signals to a planar probe facing a LPP at a distance of $9.5 \mathrm{~cm}$. (b) Typical plot of current against bias voltage (IV) for a planar probe.

thin sheath theory can be used to interpret the Langmuir probe characteristics. ${ }^{4}$

Probes with simple symmetric shapes such as spheres, infinite planes, and infinitely long cylinders are well understood and theories exist which describe their behavior. End and edge effects which could influence the probe characteristics, have received only modest attention due to the difficulty of their analyses. ${ }^{6}$ However, the collecting area of a double-sided, planar probe was investigated by Sheridan, ${ }^{7}$ using a hybrid simulation with cold, collisionless ions and Boltzmann electrons. According to their results, our probe would be considered relatively large, with minimal edge effects.

The technique of using an electrostatic probe to measure the properties of a LPP was first introduced by Koopman ${ }^{8}$ and has been used extensively since then, particularly in recent years. ${ }^{3,9}$ The probe is introduced in the plasma and biased with respect to ground. The bias voltage is swept from negative to positive voltages allowing for the measurement of ions or electrons, respectively. Both theory and experiment show, ${ }^{10}$ that for a planar probe facing the plasma flow and at negative voltages high enough to prevent the electrons with the highest thermal energies in the plasma from reaching the probe, the detected ionic current saturates. This current is proportional to the ion density and the plasma flow velocity, $v$, through the following expression:

$$
I_{\text {ion }}=e v A n_{i}
$$

where $e$ is the electron charge and $A$ is the collecting area of the probe. The above equation assumes that only singly charged ions are present. Figure 1(a) shows an example of the current to a probe, positioned $9.5 \mathrm{~cm}$ from a laser ablated target. The signal is characterized by a rapid rise in current when the ablation plasma arrives at the probe. It reaches a maximum, corresponding to the time of maximum ion flux, and then decreases as the plasma continues to expand beyond the probe and the plasma density and velocity at the probe continue to decrease. The ion energy at the time of maximum ion flux corresponds to $100 \mathrm{eV}$.

On moving the bias voltage toward positive values, the current due to electrons increases and eventually it dominates over the ion current which is not much influenced by small values of positive bias. By recording probe signals for a range of bias voltages, choosing a particular time and then plotting the measured current against bias voltage, a $I V$ characteristic is obtained. Figure 1(b) shows an example of a $I V$ characteristic. At a certain potential called the floating potential, $V_{f}$, the current due to the ions and electrons are of equal magnitude and so the measured current is zero. For bias voltages greater than $V_{f}$, the current is related to the electron energy distribution function (EEDF). For a Maxwellian distribution the measured electron current is given by

$$
I=I_{e, \text { sat }} \exp \left(\frac{V-V_{p}}{k T_{e}}\right),
$$

where $V_{p}$ is the plasma potential and $I_{e, \text { sat }}$ is the saturation electron current. The saturation electron current region is reached by further increasing the bias to values greater than the plasma potential. The saturation electron current is proportional to the density of electrons, $n_{e}$, and the average thermal velocity in the plasma, $v_{e}$, and is given by

$$
I_{e, \text { sat }}=\frac{1}{4} e v_{e} A n_{e},
$$

where $v_{e}$ is given by $\left(8 k T_{e} / \pi m_{e}\right)^{1 / 2}$. From Eq. (4), $k T_{e}$ can be readily found from a $d(\ln I) / d V$ plot of the electron-retarding region.

The ions in a LPP have a flow velocity corresponding to energies of 10-500 eV. They can still reach the probe surface, even when a positive bias of $1-3 \mathrm{~V}$ is applied. While it is possible to correct for this by subtracting the ion current from the $I V$ characteristic, ${ }^{10}$ it is not yet established that this method yields reliable values of electron temperature. We have studied the behavior of the planar probe where it is oriented so that the plasma flow is parallel to the surface of the probe. In this way the ion current due to the plasma flow can be eliminated. While the ion current for a probe oriented perpendicular to the plasma flow saturates at a bias of a few volts, this is not the case for the plasma flow along the probe surface. We have observed that the ion current increases in proportion to $V^{1 / 2}$, and have shown that the behavior can be described using a theoretical model similar to that used to describe PIII. ${ }^{11}$

In PIII, the sample to be ion implanted is immersed in a quasineutral plasma and a negative high-voltage pulse, typically $10-100 \mathrm{kV}$ is used to accelerate ions from the plasma. 


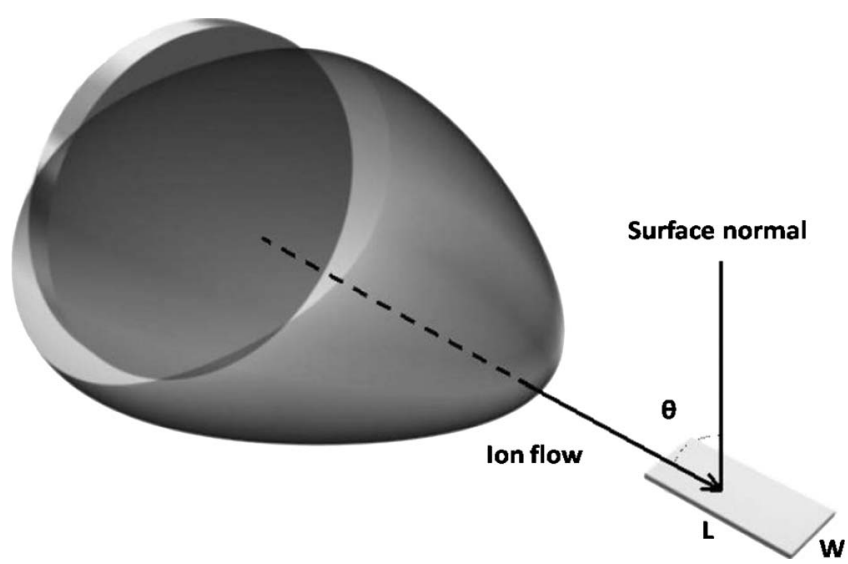

FIG. 2. The orientation of the probe relative to the plasma expansion.

When the high-voltage pulse is applied, the electrons near the surface are repelled on the time scale of the inverse electron plasma frequency, leaving behind a uniform density "matrix" sheath of ions. The electron plasma frequency is given by given by $\omega_{p e}=\sqrt{n_{e} e^{2} / \varepsilon_{0} m_{e}}$.

On the time scale of the inverse ion plasma frequency, ions within this sheath are accelerated toward the sample surface resulting in a high current pulse. The ion plasma frequency is given by given by $\omega_{p i}=\sqrt{n_{i} e^{2} / \varepsilon_{0} m_{i}}$. Subsequently the thickness of the sheath expands until a steady state Child-Langmuir sheath is formed. There is a close correspondence between applying the voltage pulse to the sample in PIII and the arrival of an element of plasma at the upstream edge of a biased planar probe lying parallel to the flow in a laser ablation plume. As a region of the plasma encounters the edge of the negatively biased probe nearest the target, the electrons are repelled and a matrix sheath is formed. The thickness, $h_{0}$ of this sheath is given by

$$
h_{0}=\left(\frac{2 \varepsilon_{0} V}{e n_{0}}\right)^{1 / 2}
$$

where $n_{0}$ is the undisturbed ion density. Ions from the matrix sheath are collected by the probe over a timescale given by

$$
\frac{v_{0}}{h_{0}}=\left(\frac{e^{2} n_{0}}{\varepsilon_{0} m_{i}}\right)^{1 / 2}=\omega_{p i}
$$

where $v_{0}=\left(2 e V / m_{i}\right)^{1 / 2}$ is the characteristic ion velocity. On a longer time scale given by

$$
t_{C} \approx\left(\frac{\sqrt{2}}{9}\right) \omega_{p i}^{-1}\left(\frac{2 V e}{k T_{e}}\right)^{3 / 4}
$$

The matrix sheath evolves into a steady state Child-law sheath, with a thickness

$$
h_{C}=h_{0}\left[\frac{2}{9} \frac{v_{0}}{v_{B}}\right]^{1 / 2} \text {. }
$$

Figure 2 shows the probe orientation relative to the plasma expansion. In this orientation, the plasma will flow parallel to the collecting surface. As an element of plasma flows along the surface, the time taken for the Child-law sheath to be established, $t_{C}$, can be shorter than the time taken to travel the length of the probe. Thus the current to the probe from that plasma element will vary in a rather complicated manner. It is possible to extend the PIII model of Riemann and Daube $^{11}$ to estimate the net current to the probe as a plasma travels the length of the probe.

The Riemann and Daube model describes the ion current peak flowing to the target for both the matrix sheath and Child-law sheath phases of ion extraction. For the matrix sheath extraction phase the model is based on a special class of simultaneous exact solutions of the ion fluid and of Poisson's equations. The model gives the following approximation for the normalized ion flux density to the target during the matrix regime expressed as a function of normalized time, $T=\omega_{p i} t$.

$$
j(T)=N(0, T) u(0, T)=\frac{1+u_{0} T+u_{0} T^{3} / 12}{\left(1+\frac{T^{2}}{2}\right)^{2}},
$$

where $N(0, T)$ is the ion density at the probe surface normalized to the undisturbed ion density, $n_{0}, u(0, T)$ is the ion velocity at the probe surface normalized to the Bohm velocity and $u_{0}=v_{0} / v_{B}=(2 \Phi)^{1 / 2}=-s_{0}$. In the sheath expansion phase the ion current density to the probe can be related to the position of the sheath boundary by the following parametric equation

$$
j(s)=1-\beta\left(1+\frac{\alpha s_{0}^{2}}{2 s^{2}}\right)\left(1-\frac{s_{\infty}^{2}}{s^{2}}\right),
$$

where $s$ is expressed in units of Debye length, $s(0)=s_{0}$ $=h_{0} / \lambda_{D}=-\sqrt{2 \Phi_{0}}$, where $\Phi_{0}=e V / k T_{e}$. It is shown ${ }^{9}$ that letting $\alpha=\frac{5}{3}$ and $\beta=1+2 s_{0} / s_{\infty}$ in Eq. (11), provides a good approximation to a full numerical model of sheath expansion, where $s_{\infty}$ is the final position of the Langmuir-Child sheath. The corresponding time $T$ when the sheath reaches $s$ is given by

$$
T(s)=T_{E}+\frac{1}{\beta}\left[s-s_{E}+s_{\infty}\left(\tanh ^{-1} \frac{s_{E}}{s_{\infty}}-\tanh ^{-1} \frac{s}{s_{\infty}}\right)\right] .
$$

$T_{E}$ is the time at which the matrix extraction phase has ended, and has been found to be $\sim 3 . s_{E}$ is the sheath thickness at this time and is approximated by $s_{E} \approx 1.6 s_{0}+3$. Figure 3(a) shows a calculation of the temporal variation of $j(T) / u_{0}$ corresponding to Eqs. (10) and (11). To find the net ion current to the probe we consider the plume to be made up of a series of slices of plasma. Their evolution in space as they travel along the probe can be equated to the evolution in time of a static plasma in contact with a probe. If $J\left(x^{\prime}\right)$ is the current density at a point $x^{\prime}$ on the probe, then the net current to the probe is given by

$$
I=\int_{0}^{L} w J\left(x^{\prime}\right) d x^{\prime}
$$

where $x^{\prime}$ is measured from the upstream edge of the probe, $L$ is the length, and $w$ is the width of the probe. This integral can be transformed to a time integral by noting that since $x^{\prime}=v t^{\prime}$, then $d x^{\prime}=v d t^{\prime}$ and since $T=\omega_{p i} t$, then $d T=\omega_{p i} d t$. Thus Eq. (13) becomes 

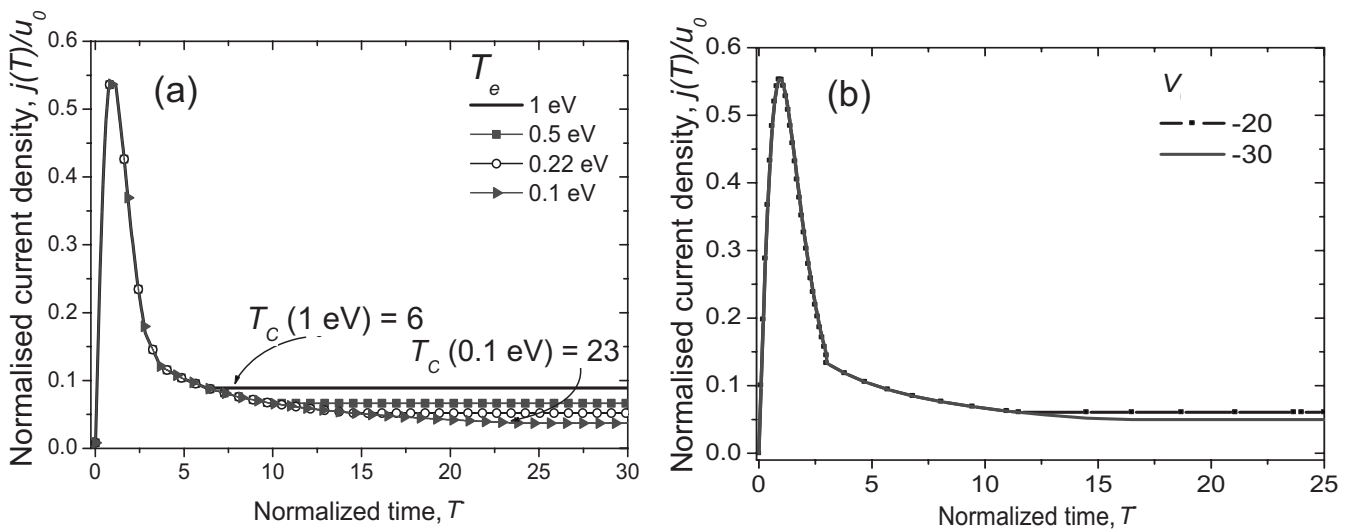

FIG. 3. (a) Temporal variation of current density for various electron temperatures. (b) The current density dependence for different bias voltages.

$$
I=\frac{w v e n_{0} v_{0}}{\omega_{p i}} \int_{0}^{T_{L}} \frac{j(T)}{u_{0}} d T,
$$

where $T_{L}$ is the normalized time taken for an element of plasma to traverse the probe. Depending on the time taken to traverse the probe, Fig. 3 can be integrated over the relevant time scale. Equation (14) is then used to calculate the current to the probe.

\section{EXPERIMENT}

A $248 \mathrm{~nm}, 26 \mathrm{~ns}, \mathrm{KrF}$ excimer laser at normal incidence, was used to ablate a rotating silver target in a vacuum chamber at $5 \times 10^{-5} \mathrm{mbar}$. The laser fluence was $1.5 \mathrm{~J} \mathrm{~cm}^{-2}$ on a $1 \times 0.5 \mathrm{~mm}^{2}$ spot. A planar Langmuir probe with dimensions $L=13 \mathrm{~mm}$ and $W=3 \mathrm{~mm}$ was placed directly in front of the target at a distance of $9.5 \mathrm{~cm}$. The probe could be rotated about an axis in the plane of the probe to investigate how the ion flux depends on angle between the plasma flow and the probe normal. To record the ion flux the probe collecting area was oriented normal to the plasma flow and biased negatively to reject electrons. Since the plasma flow in a laser ablation plume is typically supersonic, the ion current is dominated by the ion flow velocity and is given by Eq. (3). To measure $T_{e}$, the probe signals for a wide range of bias voltages were recorded. The $I V$ characteristic at a particular time was then obtained by plotting the current against the bias voltage.

\section{RESULTS AND DISCUSSION}

Figure 4 shows the bias variation of the ion current to the probe, placed at $9.5 \mathrm{~cm}$ from the target along the target normal and oriented normal to the plasma flow. Clearly a relative small negative bias of a few volts is sufficient to reject electrons and saturate the measured ion signal. It reaches a maximum that corresponds to the time of maximum ion flux at $6.5 \mu$ s and then falls as the plume continues to expand beyond the probe. As the ion flow velocity is larger than the Bohm velocity, the ion current density $J(t)$, measured on the probe is related to the ion density by the following equation:

$$
J(t)=e v n_{i} .
$$

Neglecting the acceleration time of the ablated plume, $v$ is given by the target-probe distance divided by the TOF. The neglect of the acceleration time is justified when we note that in a simulation using the isentropic gas expansion model of Anisimov et al. ${ }^{12}$ the acceleration time was found to be $50 \mathrm{~ns}$ which is small compared to the typical TOF. Figure 4 also shows the ion density derived from the probe signal obtained at a bias of $-30 \mathrm{~V}$. The ion current density $J(t)$ also yields the ion energy distribution via the following relation:

$$
\frac{d F}{d E}=J(t) \times \frac{t^{3}}{e m_{i} x^{2}},
$$

where $x$ is target-probe separation. For the distribution in Fig. 4, the mean ion energy is $110 \mathrm{eV}$, and the most probable energy is $30 \mathrm{eV}$. Figure 5(a) shows the variation of the ion signal as the probe is rotated about an axis in the plane of the probe. It can be seen from Fig. 5(b), which as the probe is rotated away from the position where it is normal to the plasma flow, the magnitude of the ion signal falls approximately as $\cos \theta$, where $\theta$ is the angle between the probe normal and the plasma flow direction. This can be understood when it is realized that the ion energy is much larger than the Bohm velocity and the magnitude of the signal varies approximately as the probe area projected in direction of the flow.

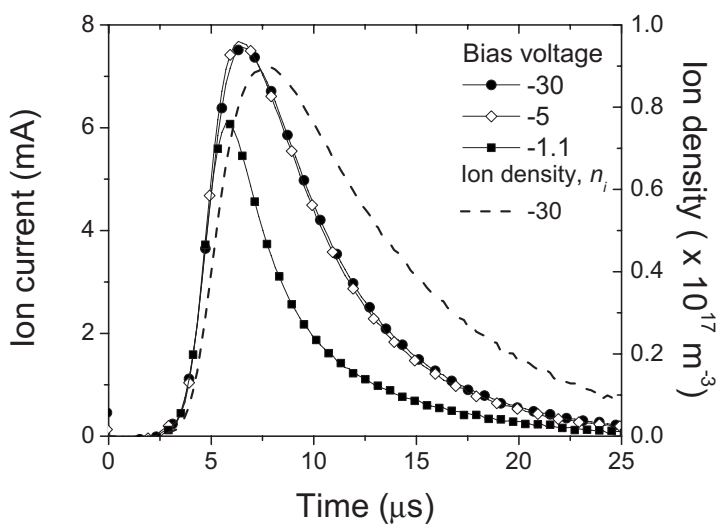

FIG. 4. Bias variation of the ion signal recorded with a probe at $9.5 \mathrm{~cm}$ from the target and oriented perpendicular to the plasma flow. 

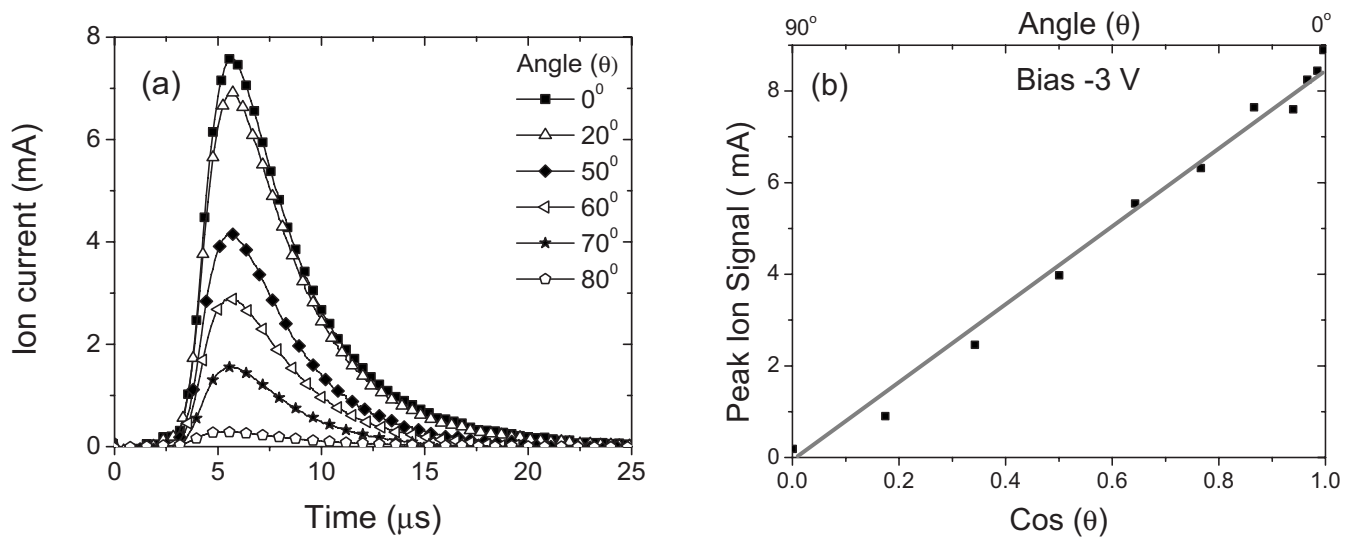

FIG. 5. (a) Variation of the ion signal as the probe is rotated in the plasma flow. (b) Peak ion current to the probe against angle.

For the time of maximum ion flux $(6.5 \mu \mathrm{s})$, the ion part of the $I V$ curves are plotted for both the parallel and perpendicular orientations in Fig. 6. For the perpendicular case the ion current does not depend on the bias voltage. However, for the parallel case, the signal does depend on the bias voltage; a log-log plot revels that the ion current varies as $V^{1 / 2}$. The temporal variation of the $T_{e}$ was measured using the probe in both orientations. For small positive bias, the net current recorded by the probe in the perpendicular position is given by the algebraic sum of the electron current and the saturated ion current since the typical ion energy is much greater than a few volts. Thus, the true electron current is obtained by subtracting the saturated ion current from the recorded current. For the probe in the parallel position there is no ion current due to the plasma flow.

The electron temperature at a particular time is obtained by taking the slope of a semilogarithmic plot of the $I V$ characteristic in the electron-retarding region of the characteristic. Figure 7 shows the temporal variation of $T_{e}$; as the ablation plume arrives at the probe position the electron temperature initially rises to a maximum value and then falls. This initial increase in temperature as the plasma arrives is consistent with the assumption that the plume expansion is both adiabatic and isentropic. ${ }^{12}$ Using Eq. (14) and the variation of ion density obtained from the probe in the perpendicular position we can estimate the ion current to the parallel probe. This was done for $T_{e}$ in the range of $0.1-1 \mathrm{eV}$.

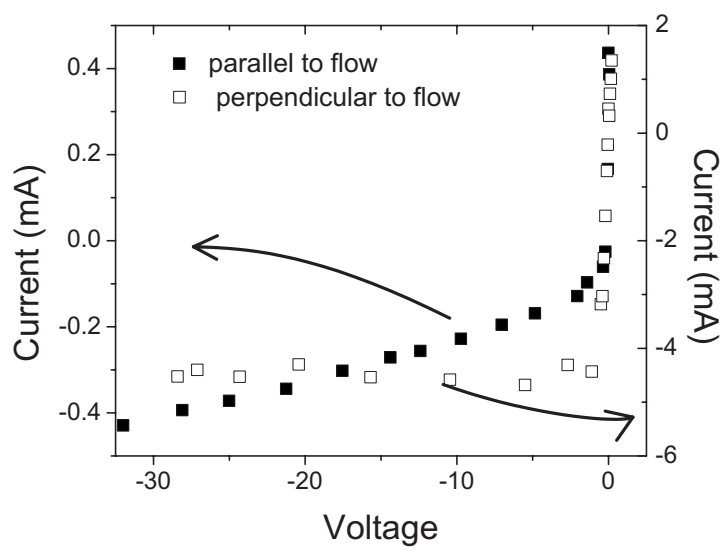

FIG. 6. Ion current variation with $\theta=0^{\circ}$ and $90^{\circ}$.
Depending on the time taken to traverse the probe ( $T=1$ for the fastest ions to $T=53$ for the slowest), $j(T) / u_{0}$ as shown in Fig. 3(a), was integrated over the relevant time scale. The calculated ion current to the parallel probe for different values of $T_{e}$ is shown in Fig. 8, together with the experimental ion signal. It seems that the best fit is produced by $T_{e}$ in the range of $0.22-0.5 \mathrm{eV}$.

From Fig. 3(a), it can be seen that for lower $T_{e}$, it takes longer for the steady state Child sheath to develop. Thus for a relatively short probe dimension $\left(T_{L}<3\right)$, the current is nearly independent of $T_{e}$. Conversely for a long probe $\left(T_{L}\right.$ $>3$ ) as used in this work, the measured ion signal depends strongly on $T_{e}$ as can be seen in Fig. 8. While the parallel probe gave $T_{e}=0.3-0.4 \mathrm{eV}$ at the time of maximum ion flux, for the same time the perpendicular probe gave $T_{e}$ $\sim 2 \mathrm{eV}$. Thus $T_{e}$ as measured by the parallel probe is consistent with the magnitude of the ion signal measured with the parallel probe while $T_{e}$ measured with perpendicular probe is not. It is not yet clear why the perpendicular probe yields a higher value for $T_{e}$ even though the correction due to the ion flux is included.

It is also evident from Fig. 3(b), that $j(T) / u_{0}$ depends weakly on the value of $V$, so that from Eq. (14), $I \propto n_{0} v_{0}$ $=\left(2 e V_{0} / m_{i}\right)^{1 / 2}$. Thus the ion signal recorded by a parallel probe is proportional to the ion density and the square root of

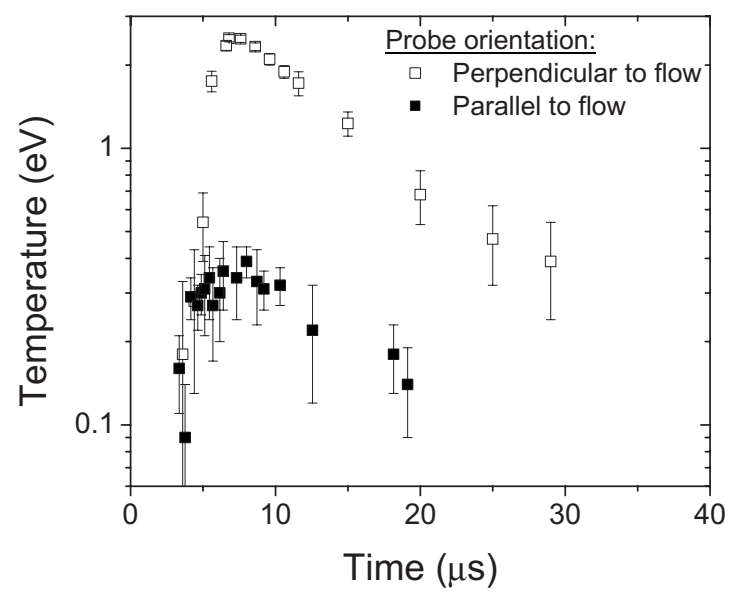

FIG. 7. The temporal variation of the electron temperature, measured using the $I V$ characteristic. 


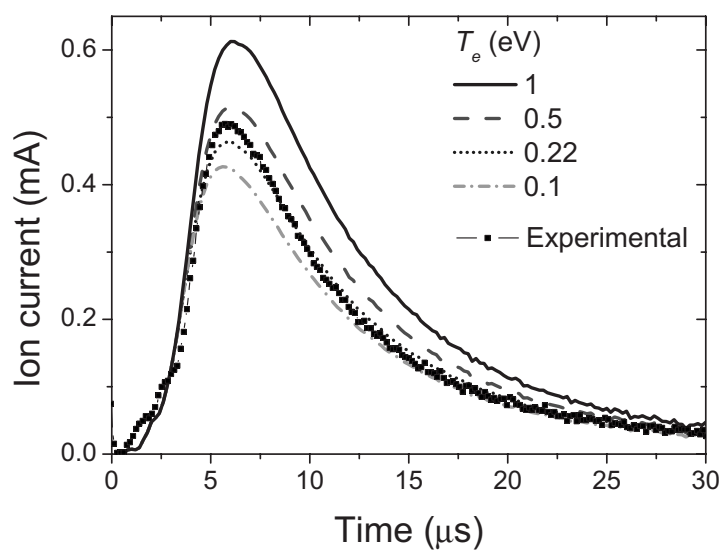

FIG. 8. The calculated current to the probe for a range of $T_{e}$, and the experimental result.

the bias voltage. It can also be seen that the current varies as $m_{i}^{-1 / 2}$ so that by comparing the ion signals from parallel and perpendicular probes it is possible to estimate the mass of the ions detected.

\section{CONCLUSION}

We have investigated the application of planar Langmuir probes in the supersonic plasma flow produced by laser ablation of a solid target in vacuum. We have shown that when the probe is normal to the flow, the ion current does not depend on the bias voltage, but rather is mainly determined by the ion flux associated with the plasma flow. Conversely when the probe surface is parallel to the plasma flow, this flow component of the ion current is turned off and the ion current is due to ion extraction caused by the probe potential relative to the plasma. We have demonstrated that it is possible to adapt an analytical model of PIII to quantitatively describe the $V^{1 / 2}$ dependence of the ion current on the bias voltage.

It seems that a probe oriented parallel to the plasma flow, where the ion current due to the plasma flow is eliminated, gives a more reliable measurement of $T_{e}$. In particular, the measured $T_{e}$ is consistent with the measured ion current which is dependent on $T_{e}$, when the time taken for an element of plasma to traverse the probe is longer than the time taken for the matrix ion sheath extraction phase. However it is not yet clear why a probe in the perpendicular orientation gives a higher value of $T_{e}$.

${ }^{1}$ T. N. Hansen, J. Schou, and J. G. Lunney, Appl. Phys. A: Mater. Sci. Process. 69, S601 (1999).

${ }^{2}$ B. Toftmann, J. Schou, and J. G. Lunney, Phys. Rev. B 67, 104101 (2003).

${ }^{3}$ B. Toftmann, J. Schou, T. N. Hansen, and J. G. Lunney, Phys. Rev. Lett. 84, 3998 (2000).

${ }^{4}$ Huddlestone, R.H. and Leonard, S.L., Plasma Diagnostic Techniques, Pure and Applied Physics Series (Academic, New York, 1965).

${ }^{5}$ R. J. Goldston and P. H. Rutherford, Introduction to Plasma Physics (Institute of Physics, Bristol, 1995).

${ }^{6}$ M. B. Hopkins and W. G. Graham, Rev. Sci. Instrum. 57, 2210 (1986).

${ }^{7}$ T. E. Sheridan, Phys. Plasmas 7, 3084 (2000).

${ }^{8}$ D. W. Koopman, Phys. Fluids 14, 1707 (1971)

${ }^{9}$ R. J. von Gutfeld and R. W. Dreyfus, Appl. Phys. Lett. 54, 1212 (1989).

${ }^{10}$ B. Doggett, C. Budtz-Joergensen, J. G. Lunney, P. Sheerin, and M. M. Turner, Appl. Surf. Sci. 247, 134 (2005).

${ }^{11}$ K. U. Riemann and T. Daube, J. Appl. Phys. 86, 1202 (1999).

${ }^{12}$ S. I. Anisimov, B. S. Luk'yanchuk, and A. Luches, Appl. Surf. Sci. 96-98, 24 (1996). 